\title{
Preface
}

\section{Hepatic Pathology: Evolving \\ Concepts in Diagnosis and \\ Pathogenesis for Medical \\ Diseases and Tumors}

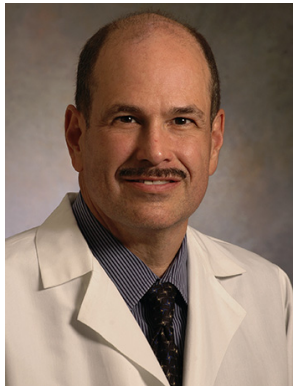

John Hart, MD

Editor

Percutaneous and transjugular liver biopsy tests have been utilized for over 50 years for the evaluation of medical liver disease and for the diagnosis of hepatic mass lesions. The increasing use of noninvasive methods for assessing liver fibrosis, coupled with the expanding utilization of directacting antiviral medications for the treatment of chronic HCV hepatitis, has clearly led to a recent decrease in the volume of liver biopsies. Nevertheless, histologic assessment of a liver biopsy specimen remains the gold standard for diagnosis of most medical liver diseases and mass lesions. Moreover, there have been exciting recent developments in our understanding of the molecular pathogenesis of many hereditary, metabolic, autoimmune, and neoplastic disorders that have made pathologic assessment of liver tissue samples more informative and clinically relevant than ever before.

In this issue, recent developments in the pathology of a variety of medical liver diseases and tumors are reviewed. There has been a significant expansion of the clinically important bacterial, fungal, parasitic, and viral infections of the liver that must be considered in an era of everincreasing global travel. The enormous differential diagnosis of granulomas and acute hepatitis now faced by the surgical pathologist requires a systematic approach for liver biopsy diagnosis. While chronic HCV hepatitis is no longer the leading indication for liver transplantation in the United States, having been supplanted by nonalcoholic steatohepatitis, there are still intriguing histologic features present in the biopsies of patients who have achieved sustained virologic response after treatment with a direct-acting antiviral agent. At the same time, our understanding of the pathogenesis of nonalcoholic steatohepatitis increases yearly in parallel with the increasing number of liver biopsies performed to assess this disorder. The pathologic features of steatohepatitis are being delineated in ever greater detail, making histologic assessment more valuable for clinical management decision making.

One of the increasingly common and important indications for liver biopsy is the assessment for possible drug-induced liver injury. As biologic agents are utilized more frequently to treat advanced malignancies, clinically significant hepatic toxicity has become a critical limiting factor in their use. The number of new medications of all types is seemingly rising every year, resulting in an ever-expanding spectrum of histologic features of drug-induced injury. At the same time, progress in identifying the key molecular events responsible for several of the common and rare hereditary and autoimmune disorders of the liver has led to a better delineation of the key histologic features necessary for their diagnosis. Hereditary cholestatic conditions due to defects in canalicular 
transporter proteins in particular are being increasingly recognized and diagnosed based on a combination of key histologic features and molecular testing performed on liver biopsy samples. Meanwhile, as our knowledge of the clinical and molecular features of autoimmune hepatitis and primary biliary cholangitis becomes more refined, the histologic spectrum of features is also becoming more detailed and clinically relevant.

Liver biopsy has always been a mainstay in the diagnosis of hepatic mass lesions, whether primary or metastatic. Tissue acquisition is even more important now as next-generation sequencing is utilized routinely to also identify mutations that can be targeted for therapeutic intervention. Our increasing knowledge of the molecular pathogenic mechanisms of hepatocellular adenoma development has led to the creation and refinement of a new classification scheme that is utilized for management decision making. New diagnostic and prognostic immunohistologic and molecular techniques are also being developed and applied in the diagnosis of hepatocellular carcinoma, fibrolamellar carcinoma, cholangiocarcinoma, and primary liver tumors with progenitor cell phenotypes. The diagnosis of lymphoproliferative disorders involving the liver also increasingly relies on the carefully considered application of an expanding selection of immunohistologic and molecular diagnostic assays.

Every practicing surgical pathologist must be prepared for frozen section consultations on liver specimens, for diagnosis of primary and metastatic tumors as well as many medical liver diseases. Donor liver biopsy frozen section evaluation is also a critical component of the decision-making process for organ suitability for transplantation and is often performed in the middle of the night without backup from colleagues. As the donor pool is expanded to meet the demand for organs, the spectrum of histologic abnormalities that needs to be evaluated in donor liver biopsies is also rising. The pathologic evaluation of posttransplant liver biopsies, after a long and dynamic period of progressive gain in experience and accumulated knowledge, has now seemingly settled into a mature field of well-defined pathologic entities. Nevertheless, as new immunosuppressive regimens are instituted and as long-term allograft survival increases, there is still an evolving understanding of the pathologic features of allograft rejection and tolerance.

This issue of Surgical Pathology Clinics is devoted to the most recent developments in each of these areas of hepatic pathology. The intent is for the reader to use this text as a resource for the most up-to-date information available for each of the 14 topics covered, with numerous high-quality and carefully curated photographs to illustrate the key features described in the text. I am grateful to all of the expert liver pathologists who have accepted the invitation to contribute. They have undertaken to provide the reader with a concise and well thought-out approach to the most common and problematic issues in hepatic pathology, and I believe they have met the challenge exceptionally well.

John Hart, MD

Department of Pathology University of Chicago Medicine MC 6101, 5841 South Maryland Avenue Chicago, IL 60637, USA

E-mail address: John.hart@uchospitals.edu 